\title{
Numerical methods for Free Boundary problems
}

\author{
L. Ferragut* \\ Keywords: Free Boundary, Numerical Methods \\ Mathematics Subject Classification (2010): 65N30, 65N50, 35Q35
} A free boundary problem is a partial differential equation to be
solved for both an unknown function $u$ and an unknown do-
main $\Omega$. The boundary $\Gamma$ of $\Omega$ or the part of it which is not
known is called a free boundary.

The simplest example is a membrane deformation in the presence of an obstacle. As many other examples the obstacle problem can be modeled by a variational inequality.

Another classic example is the melting of ice or any other substance. This is an example of a moving free boundary and is known as the Stefan Problem. Given a block of ice, one can solve the heat equation given appropriate initial and boundary conditions to determine its temperature. But, if in any region the temperature is greater than the melting point of ice, this domain will be occupied by liquid water instead. The ice/liquid interface is controlled dynamically by the solution of the PDE and it is a moving free boundary. The formulation of this type of problems can lead to a parabolic variational inequalities.
More complex free boundary problems arise in fluids mechanics when we have several non miscible fluids in contact, e.g., two phase flow in porous media, water flooding, etc. This kind of problems can be solved using level sets techniques.

In this lecture, I will give a survey concerning different numerical methods to solve free boundary problems, mainly focussing in

- Solving variational inequalities

- Adaptive Finite Element methods

- Level set methods

\section{Acknowledgements}

To all those people who once were my students and have become friends and colleagues along time.

*Institute on Fundamental Physics and Mathematics, University of Salamanca, c/ Parque, 37008 Salamanca (SPAIN). Email: ferragut@ usal.es 